\title{
Balkanologie
}

Balkanologie Revue d'études pluridisciplinaires

Vol. III, n 1 | 1999

Volume III Numéro 1

\section{Recompositions identitaires chez les musulmans de Bulgarie : entre marqueurs ethniques et religieux}

\section{Nadège Ragaru}

\section{(2) OpenEdition}

\section{Journals}

Édition électronique

URL : http://journals.openedition.org/balkanologie/290

DOI : 10.4000/balkanologie.290

ISSN : 1965-0582

Éditeur

Association française d'études sur les Balkans (Afebalk)

Édition imprimée

Date de publication : 1 juillet 1999

ISSN : 1279-7952

\section{Référence électronique}

Nadège Ragaru, «Recompositions identitaires chez les musulmans de Bulgarie : entre marqueurs ethniques et religieux », Balkanologie [En ligne], Vol. III, n 1 | 1999, mis en ligne le 03 juin 2008,

consulté le 17 décembre 2020. URL : http://journals.openedition.org/balkanologie/290 ; DOI : https:// doi.org/10.4000/balkanologie.290

Ce document a été généré automatiquement le 17 décembre 2020.

(c) Tous droits réservés 


\title{
Recompositions identitaires chez les musulmans de Bulgarie : entre marqueurs ethniques et religieux
}

\author{
Nadège Ragaru
}

1 Si la politique d'assimilation brutale des minorités musulmanes conduite par le régime Jivkov en Bulgarie dans les années 1980 a été largement commentée par des médias occidentaux prompts à prédire une escalade de violences interethniques au moment de la chute du communisme fin 1989 , on en sait en revanche fort peu sur des communautés islamiques qui représentent environ $13,1 \%$ de la population bulgare ${ }^{1}$. Certes, on se souvient qu'en 1984, le pouvoir communiste avait imposé une bulgarisation forcée des noms d'environ 800000 Turcs bulgares en vue de procéder à l'unification définitive d'une nation majoritairement chrétienne par l'éradication de la présence turque/musulmane dans le pays. Officiellement d'ailleurs, la Bulgarie ne comptait plus de Turcs, ceux-ci ayant émigré dans le cadre d'un accord bilatéral signé avec Ankara en 1968. Seuls demeuraient des descendants de Bulgares islamisés et turcisés de force pendant la période ottomane. À la faveur d'un processus de "renaissance nationale" (sic), ces derniers avaient, aux dires des autorités, redécouvert leur conscience ethnico-religieuse véritable et choisi de modifier leurs patronymes en conséquence.

2 On a également en mémoire l'exode du printemps 1989 au cours duquel s'étaient retrouvés jetés sur les routes près de 340000 musulmans de Bulgarie. Dernier avatar de la politique jivkovienne des nationalités, ce recours à l'émigration forcée, loin de faire taire les mécontentements dans les régions à population mixte, avait eu raison du peu de légitimité dont le Parti unique jouissait encore dans le pays. Au début des changements politiques, l'une des premières décisions prise par les réformateurs communistes avait d'ailleurs porté sur le rétablissement des droits des musulmans de Bulgarie.

3 Ces éléments factuels mis à part, notre connaissance des communautés musulmanes de Bulgarie demeure relativement lacunaire. Que sait-on en effet de leurs modes de vie, de 
leur rapport à la foi ou encore des lignes de clivage qui les traversent? Au cours des décennies passées, deux principaux facteurs ont influé sur les constructions identitaires de ces populations extrêmement hétérogènes. La discrimination systématique pratiquée par le régime communiste finissant figure indubitablement en première place. En deuxième position, viennent l'industrialisation et la modernisation induites par le régime communiste après 1944. C'est à la lumière de ces dynamiques que doivent être évaluer les incidences du passage à l'économie de marché sur des communautés demeurées majoritairement rurales.

Sans prétendre offrir une couverture extensive de ces transformations, la présente étude vise à esquisser une présentation des communautés musulmanes de Bulgarie respectueuse de leur diversité. Elle se voudrait en particulier attentive aux processus de redéfinition des appartenances observés depuis les débuts de la transition. L'accent y sera placé sur les relations ambiguës qu'entretiennent marqueurs ethniques et religieux, ainsi que sur les glissements qui s'opèrent des uns aux autres dans le cadre des stratégies d'intégration à la majorité chrétienne (bulgare "ethnique") ou à la minorité majoritaire (les Turcs bulgares). Depuis l'époque des indépendances nationales au XIX ${ }^{\text {ème }}$ siècle, l'histoire des Balkans se caractérise en effet par une lente et douloureuse acclimatation de la logique stato-nationale dans un espace où cinq siècles d'empire ottoman ont conféré à la religion un poids déterminant dans la construction des identités ${ }^{2}$. Au fur et à mesure que se consolidaient des États balkaniques au nationalisme d'autant plus virulent qu'il était jeune et fragile, le facteur ethnique a été appelé à jouer un rôle grandissant dans les rapports intercommunautaires. Dans le cas de la Bulgarie, ce basculement a posé avec une acuité nouvelle la question des Pomaks ces populations, bulgares par la langue et certains aspects de leur système coutumier, mais partageant avec les Turcs bulgares une foi commune -, tout comme celle des Tsiganes, divisés entre chrétiens et musulmans.

\section{Les minorités en Bulgarie selon le recensement de 1992}

5 D'après les résultats du recensement réalisé en décembre 1992, la Bulgarie compterait, sur une population de 8472317 millions d'habitants, 85,8 \% de Bulgares, 9,4\% de Turcs, $3,7 \%$ de Tsiganes et $1,1 \%$ d'autres nationalités (tableau 1). Les résultats obtenus à partir de l'identification religieuse présentent une image sensiblement différente: le pourcentage de musulmans y atteint $13,1 \%$ suite à la présence, outre les Turcs $(75,3 \%$ des musulmans de Bulgarie), de Bulgares de confession musulmane communément appelés Pomaks (13,5\%), de Tsiganes (10,5\%) ainsi que de Tatars et de Tcherkesses (tableaux 2 et 3).

Tableau 1 - Répartition de la population par groupe ethnique

\begin{tabular}{|l|l|}
\hline Groupe ethnique & Nombre \\
\hline Population totale & 8487317 \\
\hline Bulgares & 7271185 \\
\hline
\end{tabular}




\begin{tabular}{|c|c|}
\hline Turcs & 800052 \\
\hline Tsiganes & 313396 \\
\hline Russes & 17139 \\
\hline Arméniens & 13677 \\
\hline Macédoniens & 10803 \\
\hline Valaques & 5159 \\
\hline Karakatchans & 5144 \\
\hline Grecs & 4930 \\
\hline Tatars & 4515 \\
\hline Juifs & 3461 \\
\hline Roumains & 2491 \\
\hline Gagaouzes & 1478 \\
\hline Tcherkesses & 573 \\
\hline
\end{tabular}

Source : Zhelyazkova (Antonina), « Bulgaria in Transition. The Muslim Minorities », 1994, p. 9 (manuscrit).

\section{Tableau 2 - Répartition de la population par confession religieuse}

\begin{tabular}{|l|l|}
\hline Confession religieuse & Nombre \\
\hline Orthodoxe & 7247592 \\
\hline Musulman - sunnite & 1026758 \\
\hline \hline Musulman - chiite & 83537 \\
\hline Catholique & 53074 \\
\hline Protestant & 21878 \\
\hline \hline Arménien-grégorien & 9672 \\
\hline \hline Juif & 2580 \\
\hline \hline Autres & 6430 \\
\hline
\end{tabular}

Source : Zhelyazkova (Antonina), art.cit., p. 9 
Comme dans les autres pays des Balkans, la présence de musulmans en Bulgarie résulte à la fois de mouvements migratoires (avec l'installation de colons turcs après l'invasion ottomane) et de conversions à l'islam ${ }^{3}$. Des considérations stratégiques ont entraîné une forte concentration de populations turques le long de la rivière Maritsa (qui conduisait d'Andrinople/Edirne à Belgrade), dans la région de Choumen (alors une forteresse contrôlant l'accès à Silistra, le long du Danube), autour de Razgrad (ancienne forteresse romaine), ainsi que dans les Rhodopes orientales (autour de Kardjali) ${ }^{4}$. Encore aujourd'hui, les musulmans se concentrent autour de deux régions privilégiées, au nord-est du pays, dans le quadrilatère Rousse-Silistra-Varna-Choumen, et au sud, dans les montagnes des Rhodopes. Entre ces deux pôles se répartissent $80 \%$ des Turcs de Bulgarie, tandis que $90 \%$ des Pomaks habitent les Rhodopes occidentales 5 .

Tableau 3 - Répartition de la population musulmane par groupe ethnique et par langue maternelle

\begin{tabular}{|l|r|r|l|r|r|}
\hline Groupe ethnique & musulmans & $\%$ & langue maternelle & Musulmans & $\%$ \\
\hline Turc & 812067 & 75,3 & Turque & 817609 & 75,8 \\
\hline Bulgare & 142938 & 13,5 & Bulgare & 163735 & 15,2 \\
\hline Tsigane & 112923 & 10,5 & Tsigane & 89530 & 8,3 \\
\hline Autres & 10398 & 1,0 & Autre & 7542 & 0,7 \\
\hline Total & 1078326 & 100,0 & Total & 1078326 & 100,0 \\
\hline
\end{tabular}

Source : Nacionalen statističeski institut, Religijata i pălnoletnoto naselenie, Sociologičesko izsledvane, Sofia, 1993, pp. 96-97, cité in Eminov (Ali), Turkish and Other Muslim Minorities, Londres, Hurst \& Co., 1997, p. 72.

7 Les musulmans de Bulgarie se distinguent par un habitat essentiellement rural. Dans les villes, les Bulgares chrétiens représentent $91,4 \%$ de la population contre seulement $74,4 \%$ dans les campagnes. Au fil des décennies, ce différentiel entre urbains et ruraux est allé s'approfondissant, les Bulgares orthodoxes participant de façon plus active que les Turcs ou les Pomaks à l'exode rural des années 1950. Entre 1900 et 1992, le pourcentage de Bulgares (critère ethnique) habitant en ville est ainsi passé de $18,27 \%$ à $71,64 \%$, alors que, dans le même temps, la part des Turcs citadins, originellement très modeste $(1,1 \%)$, restait inférieure au tiers $(31,64 \%)^{6}$ (tableau 4). Cet ancrage rural des musulmans a pour pendant un traditionalisme que le projet de modernisation socialiste n'est pas totalement parvenu à remettre en question.

Tableau 4 - Répartition de la population en fonction du lieu d'habitation et de l'appartenance ethnique

\begin{tabular}{|l|r|r|r|r|r|r|}
\hline & 1900 & 1920 & 1934 & 1946 & 1965 & 1992 \\
\hline \multicolumn{2}{|l|}{ Total } & & & & & \\
\hline Bulgares & 2888219 & 4036056 & 5204212 & 5903580 & 7231243 & 7271185 \\
\hline
\end{tabular}




\begin{tabular}{|r|r|r|r|r|r|r|}
\hline Turcs & 531240 & 520339 & 591193 & 675500 & 780928 & 800052 \\
\hline Tsiganes & 89549 & 98451 & 149385 & 170011 & 148874 & 313396 \\
\hline Population urbaine & & & & & \\
\hline Bulgares & 526693 & 774539 & 1085005 & 1464916 & 3753153 & 5209060 \\
\hline Turcs & 5748 & 70326 & 75941 & 92881 & 138898 & 253119 \\
\hline Tsiganes & 18369 & 25050 & 40005 & 49671 & 57146 & 163896 \\
\hline Population rurale & & & & & & \\
\hline Tulgares & 2361526 & 3261516 & 4119212 & 4438664 & 3658090 & 2062125 \\
\hline Turcs & 445492 & 450013 & 515252 & 582619 & 642030 & 546933 \\
\hline Tsiganes & 7180 & 73401 & 109380 & 120340 & 91728 & 149500 \\
\hline
\end{tabular}

Source : Tomova (Ilona), The Gypsies in the Transition Period, Sofia : International Center for Minority Studies and Intercultural Relations, 1995, p. 28.

En matière de religion, il est de coutume de souligner que les musulmans sont plus croyants que les orthodoxes. En 1992, 73 \% des Turcs déclaraient avoir foi en Dieu, contre $66 \%$ des Pomaks, 59 \% des Tsiganes et $37 \%$ seulement des Bulgares orthodoxes (tableau 5). Ce constat appelle néanmoins plusieurs nuances ${ }^{7}$. En premier lieu, les résultats obtenus enregistrent de fortes variations en fonction de l'âge, de la catégorie socioprofessionnelle ou du lieu d'habitation (tableau 6). Dans les villages, ils sont ainsi $23,8 \%$ des musulmans à déclarer visiter la mosquée "fréquemment", contre seulement $10,6 \%$ dans les villes. Le facteur générationnel est encore plus discriminant puisque seuls $1,9 \%$ des jeunes de moins de 29 ans vont "fréquemment" à la mosquée contre $42 \%$ des plus de 60 ans. On notera également qu'une large majorité des jeunes musulmans reconnaît ne jamais visiter les lieux de culte (63\%). Enfin, la fréquentation des mosquées comme la prière varient de façon inversement proportionnelle au niveau d'éducation (tableaux 6 et 7).

Tableau 5 - « Croyez-vous en Dieu ? » (en pourcentage)

\begin{tabular}{|l|l|l|l|}
\hline Groupe & Oui, je crois & non, je ne crois pas & j'hésite \\
\hline Bulgare- chrétien & 37 & 35 & 28 \\
\hline Turc & 73 & 13 & 14 \\
\hline Bulgare-musulman & 66 & 15 & 19 \\
\hline Tsiganes & 59 & 22 & 19 \\
\hline
\end{tabular}

Source : « Njakoi rezultati ot izsledvaneto "Etnokulturnata situacija v Bălgariâ, 1992" », Sociologičeski Pregled, (3), 1993, p. 58. 
Tableau 6 - Fréquentation des mosquées en fonction du lieu de résidence, du genre, de l'âge et du niveau d'éducation

\begin{tabular}{|c|c|c|c|c|}
\hline & Fréquemment & de temps en temps & Rarement & Non \\
\hline Total & 20,0 & 16,1 & 19,3 & 44,7 \\
\hline \multicolumn{5}{|l|}{ Résidence } \\
\hline Ville & 10,6 & 14,3 & 15,6 & 59,5 \\
\hline Village & 23,8 & 16,8 & 20,9 & 38,5 \\
\hline \multicolumn{5}{|l|}{ Genre } \\
\hline Homme & 27,0 & 18,8 & 18,8 & 35,5 \\
\hline Femme & 12,9 & 13,4 & 20,0 & 53,8 \\
\hline \multicolumn{5}{|l|}{ Age } \\
\hline $18-29$ ans & 1,9 & 12,7 & 22,3 & 63,1 \\
\hline 30-39 ans & 16,1 & 13,4 & 20,0 & 53,8 \\
\hline $40-49$ ans & 14,5 & 11,8 & 13,2 & 60,5 \\
\hline 50-59 ans & 25,6 & 21,3 & 13,8 & 39,3 \\
\hline 60 ans et plus & 42,0 & 20,0 & 21,0 & 17,0 \\
\hline \multicolumn{5}{|l|}{ Éducation } \\
\hline Élémentaire & 33,1 & 17,2 & 16,6 & 33,1 \\
\hline Moyenne & 12,1 & 26,4 & 21,2 & 40,3 \\
\hline Lycée & 12,5 & 12,5 & 22,3 & 52,6 \\
\hline
\end{tabular}

Source : Nacionalen statističeski institut, op.cit., p.59, cité in Eminov (Ali), op.cit., p. 67.

Tableau 7 - Pratique des prières quotidiennes en fonction du lieu de résidence, du genre, de l'âge et du niveau d'éducation

\begin{tabular}{|c|c|c|c|c|}
\hline & $\begin{array}{c}\text { oui, } \\
\text { régulièrement }\end{array}$ & $\begin{array}{c}\text { oui, mais pas } \\
\text { régulièrement }\end{array}$ & $\begin{array}{c}\text { Seulement en certaines } \\
\text { occasions }\end{array}$ & $\begin{array}{c}\text { Non, } \\
\text { jamais }\end{array}$ \\
\hline Total & 41,7 & 18,3 & 23,6 & 16,3 \\
\hline Résidence & & & & \\
\hline
\end{tabular}




\begin{tabular}{|c|c|c|c|c|}
\hline Ville & 31,2 & 15,6 & 28,4 & 24,8 \\
\hline Village & 46,0 & 19,4 & 21,7 & 13,0 \\
\hline \multicolumn{5}{|l|}{ Genre } \\
\hline Homme & 39,2 & 21,3 & 21,7 & 17,6 \\
\hline Femme & 44,2 & 15,3 & 25,6 & 14,9 \\
\hline \multicolumn{5}{|l|}{ Age } \\
\hline $18-29$ ans & 16,5 & 19,4 & 37,9 & 26,2 \\
\hline $30-39$ ans & 31,5 & 21,6 & 34,2 & 12,6 \\
\hline $40-49$ ans & 32,9 & 19,7 & 25,0 & 22,4 \\
\hline $50-59$ ans & 53,2 & 20,2 & 11,7 & 14,9 \\
\hline $\begin{array}{l}60 \text { ans et } \\
\text { plus }\end{array}$ & 73,5 & 10,8 & 7,8 & 7,8 \\
\hline \multicolumn{5}{|l|}{ Éducation } \\
\hline Élémentaire & 64,8 & 14,8 & 8,5 & 11,9 \\
\hline Moyenne & 33,9 & 20,6 & 27,5 & 18,0 \\
\hline Lycée & 18,0 & 21,6 & 38,7 & 21,6 \\
\hline
\end{tabular}

Source : Institut statistique national, op.cit., p.59, cité in Eminov (Ali), op.cit., p.68.

9 Deuxième élément de relativisation, la croyance en Dieu n'équivaut nullement stricte observance des règles religieuses. En 1992, au cours d'une enquête qualitative sur la "situation ethnoculturelle en Bulgarie", seuls $30 \%$ des Pomaks et $41 \%$ des Turcs ont déclaré respecter les prescriptions du Coran en matière de consommation d'alcool et de porc $^{8}$. De la même façon, la foi ne signifie pas nécessairement connaissance du dogme. Comme le souligne Ibrahim Ialumov, directeur de l'Institut de théologie islamique de Sofia, « le niveau d'éducation religieuse est extrêmement bas. Après la fermeture des écoles coraniques à la fin des années 1940, les gens n'ont pu avoir accès à la théologie. Les imams se formaient en apprenant des plus anciens, sans pouvoir recevoir d'enseignement formel $»^{\text {. }}$.

10 Après plusieurs décennies d'intense propagande athéiste, il semblerait que le marqueur religieux en soit venu à valoir prioritairement dans les interactions entre musulmans et non-musulmans. L'appartenance à l'islam n'est jamais autant valorisée que lorsqu'il s'agit de se distinguer de la majorité orthodoxe. En revanche, au sein du groupe la foi participe surtout d'une convivialité et d'un mode de vie partagés. Témoin la définition du rituel religieux comme «meilleur moyen pour célébrer les événements du cycle de vie - baptême, mariage, enterrement " par $23 \%$ des enquêtés ou encore l'assimilation 
de la religion à un instrument de régulation des rapports familiaux (66,6\% des personnes interrogées estimant que «la religion a une influence positive sur les relations familiales »).

11 Seule une étude détaillée des diverses communautés de confession musulmane (turques, pomaques et tsiganes) nous permettra d'affiner cette brève présentation générale. Elle fera apparaître tant la pluralité des repères identitaires adoptés par chacune d'entre elles que la différenciation des stratégies déployées dans la gestion des rapports avec la majorité chrétienne.

\section{Les Turcs : une « minorité majoritaire » en voie de consolidation}

12 Les Turcs bulgares constituent aujourd'hui la minorité musulmane numériquement la plus forte et la plus compacte. À peine $2 \%$ d'entre eux sont de confession nonmusulmane (les Gagaouzes chrétiens) et seuls $2 \%$ ne parlent pas leur langue maternelle à la maison ${ }^{10}$. Estimé à 800052 lors du recensement de décembre 1992, leur nombre se situerait plutôt entre 600000 et 700 000, certains Pomaks et Tsiganes ayant à cette occasion déclaré une nationalité turque. Sur les 340000 Turcs contraints d'émigrer au printemps 1989, 150000 seraient revenus. Depuis plusieurs années, la communauté vit au rythme de mouvements migratoires, souvent saisonniers, qui épousent les fluctuations de l'économie bulgare. On soulignera que ces va-et-vient entre Bulgarie et Turquie s'inscrivent dans une histoire longue qui a fait des déplacements de population - négociés (comme en 1968) ou forcés (en 1950-1951) - un instrument de "régulation" des rapports interethniques (tableau 8).

Tableau 8 - L'émigration turque depuis la Bulgarie, 1878-1992

\begin{tabular}{|l|r|}
\hline $1878-1912$ & 350000 \\
\hline $1923-1933$ & 101507 \\
\hline $1934-1939$ & 97181 \\
\hline $1940-1949$ & 21353 \\
\hline $1950-1951$ & 154198 \\
\hline $1952-1968$ & 24 \\
\hline $1969-1978$ & 114356 \\
\hline $1979-1988$ & \\
\hline $1989-1992$ & 321800 \\
\hline \hline Total & 1160614 \\
\hline
\end{tabular}

Source : Eminov (Ali), op. cit, p. 79. 
13 Sur le plan religieux, les Turcs de Bulgarie se divisent en sunnites et alévis (environ 84000 selon le recensement de $1992^{11}$; sans doute davantage dans la mesure où nombreux sont ceux qui ne souhaitent pas afficher leur spécificité religieuse) ${ }^{12}$. Les Alévis, autrement connus sous le nom d'Aliani, d'Alijanci ou de Kizilbash-i (littéralement "têtes rouges", par référence à leur traditionnel couvre-chef rouge) vivent en communautés assez fermées dans la Dobroudja et le Deliorman (autour de Silistra, Dobritch, Koubrat, Razgrad, Rousse et Targovichte), au nord du mont Strandja ou à l'est des Rhodopes (région de Haskovo et Kardjali) ${ }^{13}$. Ils professent un islam hétérodoxe de type alévi-bektachi ${ }^{14}$. Bien qu'ils soient souvent considérés avec défiance par des sunnites les soupçonnant d'être de "mauvais croyants", ils entretiennent avec ceux-ci des relations empreintes d'une relative tolérance mutuelle. Il n'est toutefois pas rare, notamment dans l'est des Rhodopes, qu'interrogés sur leur nationalité, les Aliani préfèrent répondre en empruntant au registre du religieux : "je ne suis pas sunnite ", disent-ils alors pour marquer leur singularité par rapport au groupe dominant ${ }^{15}$. Notons enfin que les statuts adoptés par l'office du Grand mufti (Glavnoto mûftjistvo) lors de la conférence d'octobre 1997 présentent les musulmans de Bulgarie comme "sunnites de rite hanéfite", sans faire référence à l'existence d'Alévis.

Par-delà ces différences religieuses, la communauté turque est traversée par des clivages régionaux. Alors que la Dobroudja, traditionnel "grenier à blé" de la Bulgarie, est faite de larges plaines agricoles, le massif des Rhodopes ne permet que de maigres cultures vivrières (notamment de pommes de terre) à l'ouest et une monoculture de tabac à l'est. Les évolutions socio-économiques contrastées de ces deux régions depuis les débuts de la transition reflètent les inégalités en matière de dotations naturelles. La fermeture de mines qui employaient une main d'œuvre turque, pomaque ou tsigane, le démantèlement des fermes collectives ainsi que la perte de marchés à l'exportation pour le tabac ont plongé le sud de la Bulgarie dans une crise économique profonde. Le taux de chômage y avoisine les $50 \%$ et connaît des pointes à $80 \%$ dans certains villages. Les conditions de vie sont souvent dramatiques. Par contraste, la plaine du Danube offre l'image d'une zone relativement dynamique, où fleurissent les petites entreprises privées, souvent bulgaro-turques.

Ces écarts socio-économiques et socio-culturels entre Turcs du nord et du sud-est sont sensibles au niveau des perceptions mutuelles. Il n'est pas rare d'entendre des Turcs de Dobroudja se plaindre avec une pointe de paternalisme du manque de maturité de leurs frères du sud, "moins civilisés". Récemment, l'un des leaders du Mouvement des Droits et Libertés (MDL), le parti représentant les intérêts des Turcs et musulmans de Bulgarie, interrogé sur la question de savoir pourquoi son organisation avait été fondée à Varna (au bord de la Mer noire) confiait : « de toute façon, là-bas, à Kardjali, ils sont incapables de s'organiser. Ils pourraient tout juste se soulever pour les salaires; mais on ne peut pas compter sur eux pour une structure. Ça demande du travail ». Les Turcs des Rhodopes tendent, quant à eux, à se considérer comme les seuls "vrais Turcs" et à valoriser dans leur identité une authenticité que leurs homologues, plus policés, auraient perdue. Si l'on aurait tort d'absolutiser ces différences de sensibilité, on ne saurait non plus négliger le rôle qu'elles ont joué lors de la campagne de changement des noms en 1984-1985 ou encore au moment de la fondation du MDL. Les difficultés du leader du parti Ahmed Dogan, originaire de la région de Varna, à se faire accepter à Kardjali en 1991-1993 n'y sont nullement étrangères, pas plus que les réticences des habitants des Rhodopes à suivre Giuner Tahir, un enseignant né dans un village proche 
de Razgrad et devenu président du Comité d'initiative pour le renouvellement du MDL qui s'oppose à la ligne Dogan.

La mise en exergue de spécificités régionales suggère, en contrepoint, une interrogation sur la présence d'élites turques en Bulgarie. Quoique l'on ne dispose pas de statistiques fiables en la matière, il semble possible de souligner leur relative faiblesse. Depuis l'Indépendance, les exodes successifs ont décimé les leaders de la communauté. Si le régime communiste a contribué à l'émergence d'une petite classe moyenne d'instituteurs, directeurs d'école ou employés municipaux, rares sont ceux qui ont pu dépasser le niveau de la nomenklatura locale. La répression consécutive à la campagne de bulgarisation et l'exode de 1989 ont par ailleurs fortement ébranlé la communauté turque, en provoquant un brain-drain sans précédent de médecins, ingénieurs...

17 À bien des égards, la situation des Pomaks de Bulgarie se rapproche de celle des Turcs. Elle s'en distingue toutefois par la profondeur des interrogations sur l'appartenance qui agite une population longtemps en proie aux revendications concurrentes des nationalismes bulgares et turcs.

\section{Bricolages identitaires chez les Pomaks : entre bulgarité, turcité et pomacité}

En première approche, on pourrait définir les Pomaks comme des Bulgares de confession musulmane, dont les systèmes de valeurs et modes de vie mêlent référents chrétiens et musulmans. Convertis à l'islam pendant la période ottomane (principalement entre le XV ${ }^{\text {ème }}$ et le XIX ${ }^{\text {ème }}$ siècle), les Pomaks vivent en communautés fermées dans les Rhodopes de l'ouest et la vallée de la rivière Mesta en Macédoine du Pirin, ainsi que dans quelques villages dispersés autour de Lovetch (au nord du Balkan). On évalue leur nombre aux alentours de $200000-250000^{16}$. Voisins de la Grèce, les Pomaks ont été parmi les premières victimes de la militarisation à outrance de la frontière à la fin des années 1940. Utilisant les populations locales comme de véritables boucliers humains, les communistes ont envoyé dans des camps d'internement les éléments "rebelles" et déporté des villages entiers vers l'intérieur du pays afin de s'assurer de la loyauté des régions frontalières. Conjuguée à la collectivisation, cette politique a eu pour effet de détruire une économie traditionnelle qui reposait sur l'élevage et les migrations saisonnières à destination de la Grèce ${ }^{17}$. Au fur et à mesure que les Bulgares orthodoxes partaient chercher du travail en ville, les zones de peuplement à majorité pomaque ont été condamnées à un repli communautaire de plus en plus exclusif.

Après 1878, minorité religieuse à une époque où l'adoption du modèle d'État-Nation supposait une progressive "ethnicisation" des identités, les Pomaks se sont retrouvés placés en porte-à-faux dans une société pour laquelle ils faisaient figure de stigmates du "joug turc". Dans l'architecture d'un nationalisme bulgare s'articulant autour de la mémoire exacerbée des cinq siècles de domination ottomane, les Pomaks étaient ainsi voués à occuper une place privilégiée - vivantes incarnations des "conversions forcées" imputées à l'oppresseur turc. Cette centralité même explique l'acharnement avec lequel les gouvernements successifs ont tenté de faire redécouvrir leur "appartenance véritable" à des populations que l'on craignait de voir "turcisées". En contrepoint, l'hybridation des référents religieux, linguistiques et culturels a longtemps nourri, chez 
les Pomaks, le sentiment d'une double étrangeté. Parce qu'ils n'étaient reconnus comme "leurs" ni par les Bulgares orthodoxes (à cause de leur foi), ni par les Turcs (à cause de leur passé chrétien et de leur langue), leur développement a été marqué deuxième trait distinctif - par une situation d'isolement entretenant une incertitude identitaire fondamentale.

Depuis plus d'un siècle, les Pomaks font ainsi l'objet d'assignations extérieures variables au gré des impératifs nationaux des régimes en place ${ }^{18}$. Au lendemain de l'Indépendance, alors que demeure assez grande la confusion entre appartenances religieuse et ethnique, les Bulgares musulmans sont tout d'abord incités à se désigner comme Turcs et à quitter le pays dans le sillage des "maîtres" ottomans déchus. À l'époque, la conversion est en effet souvent vue comme entraînant une abdication de la nationalité. Ce n'est qu'à partir du recensement de 1905 que les Pomaks sont reconnus comme un groupe à part, et seulement dans les années 1920-1930 qu'un discours affirmant leur bulgarité est élaboré19. Sur ce point, le régime communiste ne fait que s'inscrire dans la continuité de ses prédécesseurs, et ce d'autant plus aisément que la catégorie "religion" disparaît des recensements.

21 À plusieurs reprises au cours de ce siècle, les Pomaks ont été soumis à des tentatives d'assimilation plus ou moins violentes ${ }^{20}$ : en 1912-1918 à la faveur des guerres balkaniques et du premier conflit mondial, une campagne de rechristianisation est menée dans les Rhodopes à l'initiative de l'Église orthodoxe ; après 1937, l'organisation Rodina (patrie en bulgare), fondée pour favoriser un rapprochement entre Bulgares chrétiens et musulmans, s'emploie à son tour à leur faire abdiquer noms et religion ; enfin le régime communiste devait s'y reprendre à deux fois avant de parvenir à imposer une slavisation des patronymes pomaks, une première tentative en 1962-1964 ayant dû être abandonnée devant l'ampleur des protestations. Le changement de noms entamé au début de la décennie 1970 est finalement mené à son terme en 1974. Il précède ainsi, ce qu'on tend souvent à oublier, celui des Turcs en 1984-1985.

Le terme même de Pomak ${ }^{21}$ (employé ici par commodité d'usage) est considéré comme péjoratif chez une majorité des Bulgares musulmans. Certains lui préfèrent le label de bălgari-mohamedani introduit dans les années 1930, tandis que d'autres s'appellent bălgari-mûsûlmani. Lors du recensement de 1992, des problèmes de terminologie sont apparus, qui témoignent de la persistance d'une confusion entre marqueurs d'identité. Parmi les Bulgares de confession musulmane, 70251 se sont ainsi enregistrés comme ethniquement bulgares. À la ligne "nationalité", 65546 ont préféré opter pour des réponses du type "Bulgares musulmans", "Pomaks" ou "Musulmans" (ce qui leur a valu d'être classés comme "Bulgares musulmans"). Enfin, environ 60000 Pomaks se sont identifiés comme Turcs (dont 35000 avec le turc pour langue maternelle déclarée) ${ }^{22}$. On notera enfin que, dans l'enquête sur la "situation ethnoculturelle en Bulgarie", $6 \%$ des Pomaks se sont présentés comme chrétiens ${ }^{23}$.

Ces réponses sont intéressantes à double titre. En premier lieu, par la réaction qu'elles ont suscitées au sein de la majorité orthodoxe et dans la classe politique bulgare. La désignation du turc comme langue maternelle par des populations ne la maitrisant pour l'essentiel pas a provoqué un scandale tel qu'une commission d'enquête ad hoc a dû être dépêchée sur le terrain par le Parlement. Sur la base de ses conclusions, l'Assemblée nationale devait adopter une résolution prévoyant l'annulation des résultats de Gotse Deltchev et de Iakorouda (au sud-ouest du pays), non suivie d'effets. Ce faisant, l'affaire de Iakorouda confirme la persistance du spectre de la dilution de la 
nation bulgare. Il n'est que de lire la presse quotidienne pour s'en convaincre. La peur d'une disparition des Bulgares y est régulièrement entretenue, que ce soit pour raisons démographiques (le taux d'accroissement naturel est négatif depuis plusieurs années) ou par "turcisation".

Du point de vue des Pomaks, les résultats du recensement de 1992 révèlent en second lieu les difficultés éprouvées à s'insérer dans un schéma stato-national. De façon symptomatique, au cours de l'enquête sur la "situation ethnoculturelle de la Bulgarie", à la question "qu'es-tu ?" seuls $35 \%$ des Pomaks ont répondu sur un mode "ethnique" (contre $51 \%$ des Tsiganes, $56 \%$ des Turcs et $66 \%$ des Bulgares chrétiens) ${ }^{24}$. Chez les Pomaks, la religion reste un des éléments clés de l'identité, comme en témoigne le fait qu'interrogés sur leur nationalité, une partie des Pomaks réponde simplement "musulman". Il convient néanmoins d'introduire une distinction entre la présentation de soi face à l'étranger (celui qui n'est pas Pomak) et les supports d'identification utilisés au sein même de la communauté. Si l'on en croit les recherches effectuées par Konstantinov et al., le référent religieux serait utilisé de façon privilégiée dans un contexte "hors groupe", alors qu'» une interprétation ethnique de la question de l'identité est seulement possible (...) dans une discussion interne au groupe, mais, même alors, il convient de garder en mémoire le fait qu'une description du type "turc impur" ne conduit pas automatiquement à une identification avec la majorité bulgare " (tableau 9) ${ }^{25}$. Plus encore, il semblerait que la stratification des attitudes adoptées envers les marqueurs religieux et ethniques reflète la pluralité des processus de consolidation de l'identité à l'œuvre chez les Pomaks.

Tableau 9 - Une structure d'identité à deux niveaux chez les Pomaks

\begin{tabular}{|l|l|l|l|}
\hline & Pomak & Turc & Bulgare \\
\hline \hline Premier niveau (islamique) & Pomak = musulman & Turc = musulman & Bulgare = non-musulman \\
\hline second niveau (ethnique) & Pomak = Turc non-pur & Turc & Bulgare \\
\hline
\end{tabular}

Source : Konstantinov (Yulian), Gulbrand (Alhaug), Birgit (Igla), « Names of the Bulgarian Pomaks », Nordlyd, (17), 1991, p. 27, cité in Eminov (Ali), op. cit, p. 109.

Là où certains tentent d'affirmer une identité bulgare pour se fondre dans la majorité, parfois au prix d'une reconversion à la chrétienté (dont on a pu observer plusieurs exemples au cours des années passées dans les Rhodopes), d'autres sont tentés par une identification turque. Ainsi, dans les régions de Gotse Deltchev, Iakorouda, Samovtcha, Vemingrad, Gârmen et Madan, environ un sixième des Pomaks tendrait à présenter une conscience turque, $18 \%$ d'entre eux déclarant le turc pour langue maternelle, et ce, alors que $6 \%$ seulement affirment le parler correctement ${ }^{26}$. Ce positionnement s'accompagne d'une relecture du passé expliquant l'“oubli" du turc maternel par des décennies de politique assimilationniste. Enfin, un troisième groupe essaie de sortir de cette logique bipolaire par l'affirmation d'une identité unique - "pomaque", "musulmane", "aryenne"... distincte aussi bien des Bulgares que des Turcs. Telle semblerait notamment être la perspective adoptée par Plamen Bourov, le leader du Parti démocratique du travail (non représenté au Parlement). Elle rencontrerait en particulier le soutien d'une petite classe moyenne, faite d'enseignants et d'employés de l'administration. Puisant dans un registre de traditions propres aux Rhodopes, les 
tenants de cette approche s'emploient à réactualiser des mythes des origines qui leur permettraient d'échapper à un regard dominant, faisant de leur conversion à l'islam un drame subi. Selon certains récits, les Pomaks descendraient ainsi de Thraces convertis aux VII ${ }^{\text {ème }}$ et VIII ème siècle pour se préserver des ambitions slaves et byzantines. D'autres histoires orales les présentent comme les héritiers d'émissaires arabes envoyés dans les Balkans par Mahomet. Quelle que soit l'optique retenue, cette dynamique de formation d'allégeances spécifiques présente l'avantage d'offrir aux Pomaks la possibilité de se poser en sujets autonomes de leur propre histoire.

Est-on en mesure de cerner les facteurs déterminant les types d'identité valorisés par les divers groupes de Pomaks? Bien qu'il soit encore trop tôt pour fournir des conclusions définitives, certains paramètres peuvent être isolés. Le niveau d'éducation, l'âge ou le lieu de résidence (ville/campagne) jouent assurément un rôle dans les stratégies identitaires déployées par les membres de la communauté pomaque. L'environnement immédiat des populations constitue un deuxième facteur discriminant. Dans les Rhodopes de l'est, où les Pomaks vivent entourés d'une majorité de Turcs, la tendance est plus forte à une revendication réactive de l'appartenance bulgare, laquelle s'accompagne bien souvent d'un vote en faveur du Parti Socialiste Bulgare (PSB, ex-communiste) plus ostensiblement nationaliste que son adversaire, l'Union des Forces Démocratiques (UFD). Ce choix s'explique également par le rôle imputé à certains Pomaks dans la campagne de changement des noms (1984-1985), soit en tant qu'employés dans la milice, soit parce qu'on leur proposait des avantages en termes d'emploi ou de logement en cas d'adhésion au processus de "renaissance nationale". Par contraste, c'est souvent dans les Rhodopes de l'ouest, où la population turque est plus rare, que les Pomaks sont enclins à se déclarer Turcs.

Par ailleurs, opter pour l'un ou l'autre des grands groupes "ethniques" fournit aux Pomaks l'occasion non seulement de mettre fin à une pesante situation de tiraillement identitaire, mais également d'espérer une promotion sociale. Largement dépourvue d'élites, la communauté pomaque connaît en effet une pauvreté endémique, aggravée depuis les débuts de la transition. Son mode de vie montagnard et rural n'augure aucune amélioration prochaine de son pouvoir d'achat. Or, pour les Bulgares musulmans qui ont choisi de conserver les noms slavisés octroyés de force sous le régime communiste, l'intégration à la majorité bulgare peut être réalisée de manière relativement souple, en particulier lorsqu'ils se sont établis en ville. Bien que s'insérant dans un contexte spécifique, l'adhésion à la communauté turque présente des avantages similaires. Depuis plusieurs décennies, on observe une tendance à l'alignement progressif de l'ethnicité sur la religion, dont on oublie souvent qu'il fut originellement encouragé par le régime communiste jusqu'aux années 1950. En l'occurrence, le caractère compact de la minorité turque comme la reconnaissance qu'elle a pu obtenir grâce au MDL lui confèrent une force d'attraction particulière. La proximité de la Turquie, où certains Pomaks désireux émigrèrent en 1989, constitue un atout supplémentaire, dans la mesure où les Pomaks ne disposent pas d'un "État parrain" susceptible de les soutenir ${ }^{27}$.

\section{Les Tsiganes : l'ostracisme d'une communauté divisée}

La fluidité des identités décrite précédemment caractérise a fortiori les Tsiganes de Bulgarie, une minorité économiquement pauvre, socialement marginalisée et 
confrontée à un racisme endémique (tableau 10). La discrimination dont les Tsiganes sont victimes explique les réticences de nombre d'entre eux à s'identifier comme tels lors des recensements. Évalué en 1992 à 313 396, leur nombre avoisinerait plutôt les 500000 à $600000^{28}$. Cette différence entre statistiques officielles et données d'experts se répercute de façon décisive sur la distribution des confessions religieuses au sein de la communauté. Si l'on en croit les résultats du dernier recensement, 60,4\% d'entre eux seraient chrétiens et 39,2\% musulmans. Ces chiffres semblent toutefois biaisés par le fait qu'une partie des Gitans musulmans s'identifie comme turque ${ }^{29}$.

Tableau 10 - Stéréotypes anti-tsiganes chez les Bulgares, Turcs et Pomaks

\begin{tabular}{|l|l|l|l|l|l|}
\hline Bulgares & $\%$ & Turcs & $\%$ & Pomaks & $\%$ \\
\hline voleurs & 99 & voleurs & 99 & voleurs & 90 \\
\hline insouciants & 94 & paresseux & 88 & musiciens/artistes & 84 \\
\hline paresseux & 93 & insouciants & 87 & paresseux & 84 \\
\hline indisciplinés & 86 & indisciplinés & 79 & indisciplinés & 82 \\
\hline musiciens/artistes & 84 & musiciens/artistes & 77 & insouciants & 78 \\
\hline déloyaux/traîtres & 65 & ingrats & 67 & débauchés & 63 \\
\hline à l'esprit de clan & 65 & débauchés & 60 & ingrats & 51 \\
\hline débauchés & 63 & cruels & 60 & déloyaux/traîtres & 52 \\
\hline ingrats & 62 & déloyaux/traîtres & 52 & cruels & pauvres \\
\hline
\end{tabular}

Source : Tomova (Ilona), «Etničeskite Stereotipi », in Krasimir Kanev, éd., Etnokulturnata situacija v Bălgarija, Sofia : Access / BBN, 1993, p. 37.

Installés en Bulgarie au cours du Moyen Âge, les Tsiganes ont été relégués au bas de l'échelle sociale dès la conquête ottomane ${ }^{30}$. Le système d'imposition de l'empire introduisant une distinction entre Tsiganes musulmans nomades et Tsiganes chrétiens sédentarisés, certains auraient très tôt embrassé l'islam dans l'espoir d'améliorer leurs conditions de vie et de rehausser leur prestige social. En dépit de ces stratégies d'adaptation, les gitans ont connu une marginalisation croissante après le XVII ème siècle, au fur et à mesure que se répandaient des stéréotypes négatifs à leur égard. À la faveur de l'urbanisation de la Bulgarie au XX ${ }^{\text {ème }}$ siècle, la part des Tsiganes citadins est passée de 20,5\% en 1900 à $52 \%$ en 1992, ce qui les place à mi-chemin entre les Turcs et les Bulgares orthodoxes. Dans les grandes et moyennes villes (Sofia, Plovdiv ou Sliven, par exemple), ils vivent dans des ghettos insalubres et surpeuplés. Désireux de sédentariser une population aux traditions semi-nomadiques, le régime communiste a commandé la construction de nouveaux quartiers tsiganes après 1958, généralement en marge des villes. En l'absence de plans ultérieurs d'aménagement du territoire, ceux-ci 
se sont révélés impropres à accommoder une population jeune à forte croissance démographique (tableau 11).

Tableau 11 - Structure par âge des principaux groupes ethniques (en pourcentage)

\begin{tabular}{|l|c|c|c|}
\hline Age & Bulgares & Turcs & Tsiganes \\
\hline $0-29$ & 37,3 & 51,4 & 66,0 \\
\hline $30-59$ & 40,5 & 37,0 & 28,9 \\
\hline 60 et plus & 22,2 & 11,6 & 5,1 \\
\hline
\end{tabular}

Source : Tomova (Ilona), The Gypsies (op.cit.), p.30.

30 À l'époque communiste, la situation des ruraux était relativement plus favorable que celle des citadins, la collectivisation des terres et les besoins croissants en main d'œuvre leur assurant un emploi régulier dans l'agriculture ou dans des complexes industriels installés à la lisière des préfectures de province. Cette embauche compensait la disparition progressive d'artisanats et de métiers traditionnels (maréchaux-ferrants, forgerons, fabricants de paniers, chaussures ou brosses, dresseurs d'animaux...) rendus superfétatoires par la modernisation. Depuis le démantèlement des coopératives et la restitution des terres, la situation de cette frange de la population s'est fortement détériorée, la plupart des paysans tsiganes ne disposant pas de terres propres. Si l'on ajoute à cela le fait qu'en cas de licenciement les Tsiganes sont les premiers à être renvoyés et que les nouveaux patrons du secteur privé semblent réticents à les embaucher, on ne s'étonnera guère que le taux de chômage au sein de la communauté tsigane dépasse les trois-quarts $(76 \%)^{31}$. Dans ces conditions, la survie du groupe passe soit par un retranchement dans les montagnes où, se livrant à la cueillette de fruits sauvages et au ramassage d'escargots destinés à l'exportation, les Tsiganes représentent une concurrence inopportune pour les populations locales, soit par une migration vers les villes. Là-bas, les nouveaux arrivants vivent généralement d'aides sociales (largement réduites depuis le changement de régime) ou d'activités socialement condamnées (mendicité, vol, prostitution, ....) ${ }^{32}$. Certains d'entre eux, notamment dans le nord-est du pays, misent également sur un commerce plus ou moins légal avec la Turquie voisine ${ }^{33}$.

31 Présentant un profil démographique typique des sociétés pré-modernes (taux de fécondité élevé, mariages précoces et durée de vie limitée), les Tsiganes constituent également le groupe au sein duquel le degré d'alphabétisation et le niveau d'éducation sont les plus bas (tableau 12). Faute d'une alimentation quantitativement et qualitativement adaptée, ainsi que d'un accès régulier aux services de santé (désormais payants), ils sont par ailleurs impuissants à endiguer le développement de maladies chroniques comme la multiplication des malformations chez les enfants. Alors que la crise économique d'après 1989 a entraîné une chute du nombre des naissances dans l'ensemble de la société bulgare, certains des Tsiganes les plus défavorisés continuent à privilégier un modèle de famille nombreuse qui les voue à une marginalité croissante, mêlant alcoolisme et violences domestiques ${ }^{34}$. 
Tableau 12 - Structure des groupes ethniques en fonction du niveau d'éducation

\begin{tabular}{|l|c|c|c|}
\hline Éducation & Bulgares & Turcs & Tsiganes \\
\hline \hline Supérieure & 20,2 & 2,0 & 0,9 \\
\hline \hline Secondaire & 54,0 & 24,6 & 7,8 \\
\hline Élémentaire & 22,6 & 55,0 & 46,2 \\
\hline Primaire & 3,0 & 16,0 & 36,7 \\
\hline \hline Illettrés & 0,2 & 2,3 & 8,5 \\
\hline
\end{tabular}

Source: Tomova (Ilona), The Gypsies (op.cit.), p. 59.

En réaction à l'opprobre sociale à laquelle ils sont confrontés, les Tsiganes oscillent souvent entre la dissimulation de l'identité et l'assimilation à d'autres communautés (tableau 13). Quatre types d'auto-identification sont généralement privilégiés : si deuxtiers des Tsiganes se reconnaissent comme tels, environ $22 \%$ ont une conscience turque, $10 \%$ bulgare et $5 \%$ valaque $^{35}$. Les Tsiganes-turcs, que l'on rencontre notamment à Plovdiv, Pazardjik, Veliko Turnovo, Kardjali, Haskovo, Rousse ou Varna ainsi que dans des localités mixtes bulgaro-turques, sont généralement musulmans. Certains d'entre eux ont le turc pour langue maternelle. Tous ne sont pas pour autant acceptés par leur nouvelle communauté d'appartenance. Si les agiupti ont réussi à se faire reconnaître comme Turcs dans la région de Madan et comme Bulgares musulmans à Smolian, il n'est pas rare que les tentatives de ré-identification rencontrent la méfiance de Turcs bulgares qui soulignent les différences existant en termes de mode de vie (notamment l'attitude envers le vol) et qui jugent approximative leur maîtrise de la langue. L'insertion dans la majorité bulgare est, quant à elle, le fait des djurevtsi ou des Tsiganes les plus éduqués des grands centres urbains, à Sofia ou Sliven, par exemple. C'est sans doute dans cette dernière ville que les gitans, employés dès le $\mathrm{XIX}^{\text {ème }}$ siècle dans les toutes premières industries textiles du pays, sont le mieux intégrés à la société locale. Elle a d'ailleurs fourni l'essentiel des médecins, avocats ou écrivains tsiganes. Enfin, les Tsiganes valaques vivent pour l'essentiel dans le nord-est du pays, où ils ont longtemps été employés dans l'agriculture.

Parmi les facteurs dont dépend le succès de ces entreprises de redéfinition des appartenances, on peut souligner la nature de l'habitat (dispersé vs. en ghetto) et le degré de réussite sociale. Ce travail sur l'“ethnicité" est également influencé par l'existence, chez les Tsiganes, d'une architecture identitaire où la langue et la religion jouent un rôle différent de celui observé parmi les Bulgares chrétiens ou les Turcs. Si la plupart des Tsiganes déclarent parler le romani, les cas de bilinguisme ou de trilinguisme sont ainsi monnaie courante, et le domicile ne constitue pas nécessairement le lieu privilégié de l'expression en romani (tableau 14). De la même façon, sur moyenne ou longue période, les Tsiganes tendent à adopter la religion qui prévaut dans leur milieu environnant, en partie afin de s'y faire mieux accepter. C'est alors la nouvelle confession qui détermine l'appartenance ethnique. Pour autant, au quotidien le respect des préceptes religieux est rarement strict chez les Tsiganes, le niveau d'éducation religieuse y est assez bas et les cas d'hybridation des cultes 
fréquents. Non que la croyance occupe une place modeste dans leur existence, mais elle mêle aux principes monothéistes tout un ensemble d'éléments pré-chrétiens et de superstitions.

Tableau 14 - «Quelle langue parlez-vous en général à la maison ? » (en pourcentage)

\begin{tabular}{|l|l|l|}
\hline & oui (en \%) & non (en \%) \\
\hline Bulgare & 51,0 & 46,0 \\
\hline \hline Turc & 34,0 & 63,0 \\
\hline \hline Romani & 67,0 & 33,0 \\
\hline \hline Autre & 0,4 & 98,0 \\
\hline
\end{tabular}

Source : « Njakoi rezultati » (art.cit.), p. 57. insister sur l'hétérogénéité de la communauté tsigane. Les deux principaux ensembles (chrétiens et musulmans) se subdivisent en multiples sous-groupes suivant la profession, le mode de vie (nomade ou sédentarisé), les dialectes, les origines, la région ou ville d'appartenance, ou encore les relations lignagères. Iltchev et Perry distinguent ainsi les jerlii, descendants des premiers Tsiganes installés dans les Balkans, des kardarachi, descendants de populations nomades sédentarisées dans les années $1950^{36}$. Vivant en cercle fermé, les kardarachi comptent parmi les Tsiganes les plus riches de Bulgarie, leur fortune provenant souvent d'activités mafieuses (notamment de la prostitution) et cultivent un sentiment de supériorité. E.Marouchiakova et V.Popov identifient quant à eux pas moins de dix groupes distincts ${ }^{37}$. Comme l'explique Ilona Tomova, "les Tsiganes sont seulement une "communauté" dans la mesure où les "autres" sont concernés $»^{38}$. Cette extrême fragmentation fait obstacle à la constitution d'organisations ou de partis en mesure de prendre fait et cause pour une minorité lourdement discriminée.

Pour toutes ces communautés turques, pomaques et tsiganes, dont on a tenté de retracer les systèmes d'identification et les stratégies d'intégration à la société bulgare, quels sont exactement les effets du passage à la démocratie et au capitalisme, et plus particulièrement quelles sont les réponses qui ont été apportées à l'effondrement économique des 1990-1992 et de 1996 ? C'est ce que nous allons maintenant tenter de voir brièvement.

\section{Les effets du passage à l'économie de marché : un défi identitaire?}

On sait que la Bulgarie est l'un des pays d'Europe de l'est où le coût social des changements économiques a été le plus élevé. L’ancien système communiste y laissait en héritage une dette extérieure colossale ${ }^{39}$, une forte dépendance énergétique envers l'Union soviétique et une spécialisation industrielle très défavorable dans le cadre du $\operatorname{COMECON}^{40}$. Les incertitudes de la transition politique, l'absence de consensus sur les 
réformes et les retards pris dans la privatisation comme dans la restructuration du secteur bancaire ont conduit à un effondrement dramatique des niveaux de vie (près de $50 \%$ entre 1989 et $1995^{41}$ ), ainsi qu'à un dérapage hyperinflationniste à l'hiver 1996-1997. Depuis l'été 1998, Sofia vit sous un directoire monétaire que supervise le Fonds Monétaire International (FMI).

Cette situation socio-économique désespérée s'est répercutée sur les minorités musulmanes de Bulgarie à travers la crise du secteur agricole, la fermeture de centres industriels des zones mixtes, la réduction drastique des programmes sociaux et enfin la diminution des subventions au tabac - une production essentielle à l'économie des Rhodopes. La question de la terre est sans doute la plus sensible. Confronté à un choix entre privatisation et restitution des biens nationalisés par le pouvoir communiste à la fin des années 1940, le gouvernement Dimitrov (novembre 1991-octobre 1992) avait décidé de donner la priorité à la seconde, dans l'espoir de reconstituer une classe de petits propriétaires terriens qui lui servirait de base électorale. Parallèlement, il ordonnait un rapide démantèlement des coopératives communistes. Ce volontarisme n'a toutefois pas suffi à éviter la désorganisation du secteur agricole. Faute de cadastre pour la période d'avant-guerre et suite au fort morcellement de la propriété, la restitution a procédé avec une lenteur aggravée par l'insuffisance des mesures d'aide à la restructuration de l'agriculture (réglementation des prix, des exportations, du marché de la vente et de la location des terres, ... ${ }^{42}$. En septembre 1996, seulement $54 \%$ des terres avaient été restitués, dont $18 \%$ dans leurs limites réelles et $6 \%$ avec l'octroi de véritables titres de propriété ${ }^{43}$. Les chiffres sont encore plus bas à Kardjali ( $\left.25 \%\right)$, région montagneuse. Notons que l'instabilité politique chronique dont souffre la Bulgarie depuis 1990 (avec quatre élections législatives et sept gouvernements en l'espace de sept ans) n'a pas non plus facilité le redressement d'un secteur stratégique. À leur retour au pouvoir en décembre 1994, les anciens communistes imposaient ainsi une redéfinition des priorités du gouvernement bulgare qui prévoyait la reconstitution des coopératives au détriment de l'accession à la propriété privée.

Chez les musulmans, la restitution est problématique à un double titre: en premier lieu, rares sont les Turcs habilités à en bénéficier dans la mesure où ils étaient avantguerre moins souvent propriétaires que les Bulgares orthodoxes. L'exode rural des années 1950 ayant drainé la population turque vers les villages délaissés par leurs anciens habitants orthodoxes, la question des paysans sans terres se pose avec acuité ${ }^{44}$. En second lieu, le nombre d'héritiers entre qui les terres doivent être partagées est généralement plus élevé chez les musulmans ${ }^{45}$. Au résultat, les parcelles atteignent un degré de fragmentation parfois tel que les exploitations agricoles sont vouées à la faillite. Enfin, là où les nouveaux propriétaires bulgares mettent leurs terres en location, Turcs, Pomaks et Tsiganes ne disposent souvent pas des ressources suffisantes pour signer des baux, encore moins pour acheter les machines et engrais indispensables. En vue de contenir une situation potentiellement explosive, les autorités bulgares ont certes octroyé à chaque municipalité un fonds de terres destiné à un usage locatif. Mais jusqu'à présent ces mesures parcellaires ne sont parvenues ni à endiguer la montée du chômage agricole, ni à prévenir la désertion des villages.

La situation n'est guère plus prometteuse dans le secteur du tabac. On se souvient que, dans les années 1980, la Bulgarie était le troisième producteur de tabac en Europe (après la Turquie et l'Italie) et qu'elle occupait le deuxième rang mondial en matière d'exportation de tabac et de cigarettes ${ }^{46}$. Si la crise du tabac ne date pas de la chute du 
communisme, cette dernière achève de faire perdre à Sofia sa prééminence sur les marchés internationaux. Il suffit, pour mesurer l'ampleur du recul, de considérer le cas de Kardjali, où la production est passée de 25103 tonnes en 1987 à 13677 tonnes en 1989 pour atteindre son niveau le plus bas en 1995 avec 3700 tonnes $^{47}$. Pour autant, le puissant monopole Bulgartabac n'a pas cessé de dégager des bénéfices, mais les salaires des petits producteurs sont rarement en correspondance avec les profits qu'ils génèrent. Le cycle de production, qui suppose la gestion des décalages entre période d'ensemencement et versement des salaires les place dans une situation de grande précarité, et ce d'autant plus que les incertitudes de la transition économique nuisent à la qualité de leurs anticipations.

Dans ces conditions, quelles sont les réponses apportées par les populations musulmanes de Bulgarie à la crise ? On remarquera tout d'abord que les femmes figurent au premier rang des victimes du chômage, elles qui représentaient environ $60 \%$ de la main d'œuvre dans le secteur du tabac et presque autant dans des industries légères (notamment textiles) qui tournent désormais à capacité réduite. Certaines ont pu retrouver un emploi précaire dans de petits ateliers de confection ouverts par des entrepreneurs grecs. D'autres ont choisi de se retirer de la vie active et se consacrent à l'entretien d'un lopin de terre individuel. L'expansion du secteur privé (commerce, construction, textile) fournirait par ailleurs une source de revenu appréciable si elle ne se heurtait au manque de capitaux ainsi qu'à la faiblesse des infrastructures en zone turque. Les nouvelles entreprises sont souvent de taille modeste et dégagent des bénéfices limités. À titre d'exemple, en 1994 les 4076 entreprises privées de Kardjali fournissaient seulement $5,4 \%$ du revenu brut de la ville ${ }^{48}$. Afin de subvenir aux besoins de leurs familles, il n'est pas rare que les hommes partent chercher du travail dans les grandes villes (à Bourgas, Plovdiv, Sofia ou Varna pour le secteur de la construction, par exemple) ou à l'étranger (Turquie, Allemagne, ...). Dans l'interlude, Turcs, Pomaks et Tsiganes vivent d'aides sociales ou d'expédients.

41 Même s'il est encore trop tôt pour dresser un bilan définitif, on ne peut manquer d'être frappé par les effets de recomposition des communautés musulmanes qui découlent de ces stratégies de survie. Il semblerait tout d'abord que les mouvements migratoires et la restructuration de l'emploi soient en train d'encourager un éclatement de la cellule familiale. Prenons pour illustration le cas des employés dans la construction. En règle générale, le bâtiment paye plutôt bien. Il a cependant pour contrepartie un éloignement prolongé des maris qui n'ont ni le temps ni les moyens de faire des allerretour entre ville et campagne. La dégradation des services publics contribue significativement à cet état de fait, dans la mesure où, après 1989, les lignes d'autobus non rentables ont été fermées. Comme la hausse des prix de l'énergie a contraint à remiser les voitures au garage, il n'est pas rare que les époux restent séparés pendant plusieurs mois, le temps d'un chantier. Là encore, la situation des femmes est particulièrement dramatique. À l'époque communiste, elles cumulaient souvent emploi en ville (dans l'industrie légère ou l'administration) et travail agricole. Pour les nouvelles chômeuses, le retour au foyer signifie donc une distension des liens avec l'univers citadin ainsi qu'un repli forcé sur l'espace villageois. Ce faisant, l'un des principaux "acquis" du système communiste se trouve remis en cause - à savoir l'égalitarisation (relative) des rapports hommes / femmes fondé sur une banalisation du travail féminin. À moyen terme, il y a fort à parier que, dans des communautés au système de valeurs déjà fortement ébranlé par le passage au capitalisme, la 
transformation des modes de vie entraînera une redéfinition traditionaliste de la place des femmes dans la société.

Les observations dont nous disposons demeurent toutefois embryonnaires. Dia Anagnostou a tenté de mesurer les effets de la crise socio-économique sur les systèmes de valeur et la religiosité des musulmans de Bulgarie dans deux provinces, à Razgrad et Kardjali. Elle conclut qu'» il n'est pas possible de postuler une relation directe entre, respectivement, développement ou crise socio-économique et déclin ou réveil culturel» car "les changements socio-économiques sont contradictoires plutôt qu'uniformes" et «le sens et le contenu des liens religieux-culturels varient en fonction de la façon dont les différents systèmes sociaux, politiques et économiques sont organisés $»^{49}$. En dernier ressort, le principe de causalité serait politique :

en Bulgarie (...), les changements socio-économiques ont jeté les bases d'une identité religieuse potentiellement plus traditionnelle mais ne déterminent pas - et ne peuvent déterminer - qu'il en sera ainsi. L'activité et l'éducation religieuse pourraient promouvoir les choses dans ce sens, mais d'autres facteurs pourraient inverser ce processus. (...) Afin de déterminer cela, il faut également explorer les changements politiques dans ou entre les pays ${ }^{50}$.

Quoi qu'il en soit, la reconstruction des solidarités familiales devrait peser de façon décisive sur les évolutions des communautés musulmanes de Bulgarie.

Un autre facteur est appelé à influencer ces processus. Là où la politique répressive des années 1980 avait renforcé la cohésion de la communauté turque, les transformations économiques de l'après-communisme sont en train de favoriser l'émergence de nouveaux clivages internes. Une première division sépare désormais ceux qui sont parvenus à s'adapter aux changements (en établissant, par exemple, de petites firmes spécialisées dans le commerce) de ceux qui demeurent attachés au principe de la coopérative (craignant, pour le cas où ils loueraient leurs services, d'être exploités aussi bien par leurs patrons turcs que bulgares) ${ }^{51}$. L'accès à l'aide en provenance de Turquie constitue une seconde variable discriminante. La communauté turque est ensuite divisée politiquement : si la majorité des Turcs bulgares reste fidèle au Mouvement des Droits et Libertés, certains privilégient dorénavant l'Union des Forces Démocratiques (centre-droit), d'autres sont tentés par les mouvements agrariens, d'autres enfin votent pour le Parti socialiste en souvenir de l'époque heureuse où le système communiste leur garantissait emploi et sécurité. Il n'est jusqu'à la mémoire de la période de changement des noms qui ne soit gérée de manière différenciée. Faut-il rappeler que tous les Turcs n'ont pas repris leurs anciens noms turco-arabes? Certains, notamment parmi les jeunes, auraient préféré conserver les patronymes bulgares qui leur avaient été attribués en 1984-1985 " parce qu'ils pensent que leurs noms slaves les autoriseront à voyager à travers le monde sans être inquiétés ${ }^{52}$. Face à la crise, le désir de maximiser les opportunités professionnelles suscite ainsi des choix identitaires contrastés ${ }^{33}$.

\section{Conclusion}

Au terme de cette présentation des musulmans de Bulgarie, on espère être parvenu à souligner la prégnance des lignes de clivage qui passent aussi bien entre communautés turque, pomaque et tsigane qu'en leur sein. Certains sont alévis, d'autres sunnites. On rencontre des turcophones comme des bulgarophones ou des populations parlant le romani. Les rapports au religieux vont d'une ferveur assez intense chez certains Turcs 
et Pomaks à une indifférence proche de l'agnosticisme parmi les citadins de moins de 30 ans. À elle seule, cette diversité devrait suffire à remettre en cause les lectures de l'islam balkanique inspirées de la "théorie" de la "transversale verte", et qui le présentent comme un tout uniforme prêt à se liguer contre les majorités chrétiennes de la péninsule.

La complexité des systèmes d'allégeances rencontrés chez les populations islamiques de Bulgarie a également pour corollaire une grande variété de réponses aux défis de la transition. Souvent, celles-ci passent par la revalorisation sélective de composantes identitaires sujettes à d'importantes évolutions sur moyen terme. Que l'on vise une assimilation à la majorité chrétienne ou un rapprochement avec les Turcs fondé sur la foi commune, la multiplication des bricolages identitaires et leur utilisation comme mode d'ajustement à un environnement changeant constituent des phénomènes qui mériteraient une attention plus méticuleuse.

Certes, dans un pays relativement jeune comme la Bulgarie (indépendante depuis 1878), la malléabilité des constructions identitaires se comprend aisément. Cette fluidité a d'ailleurs sans doute facilité au cours du siècle qui s'achève la coexistence pacifique entre des communautés chrétiennes et musulmanes que l'adhésion de la Bulgarie à une vision centralisatrice de l'État-Nation aurait pu vouer à une confrontation durable. Pourtant, à l'heure où Sofia se trouve engagée dans des réformes économiques qui remettent douloureusement en cause les acquis sociaux de l'ère communiste, on ne saurait manquer de s'inquiéter de la politisation éventuelle de remaniements identitaires entrepris. La polémique suscitée en 1993 par l'auto-identification de certains Pomaks comme Turcs dans la région de Gotse Deltchev en apporte une illustration éloquente. Dans un contexte régional des plus instables, il pourrait en effet être tentant, pour les dirigeants politiques bulgares comme pour les leaders des communautés musulmanes, de manier à des fins d'auto-reproduction les langages conflictuels l'identité.

\section{NOTES}

1. Chiffres du recensement de décembre 1992. Voir ci-après la répartition détaillée des diverses populations de confession islamiques.

2. L'empire était organisé selon le système du millet, qui conférait aux communautés religieuses une large autonomie en matière d'administration locale. Pour de plus amples détails, voir Castellan (Georges), Histoire des Balkans, XIV ème-XX'ème siècle, Paris : Fayard, 1991, p. 118 et suiv.

3. La périodisation et l'analyse des modalités de la conversion à l'Islam en Bulgarie fait encore l'objet d'importantes controverses. Pour une étude critique des diverses historiographies balkaniques sur cette question, on se reportera à l'excellent Želiazkova (Antonina), «Formiraneto na mjusjulmanskite obštnosti i kompleksite na balkanskite istoriografii » (La formation des communautés musulmanes et les complexes des historiographies balkaniques), in Želiazkova (Antonina), ed., Mûsulmanskite na Balkanite i v Bălgarija (Les communautés musulmanes dans les Balkans et en Bulgarie), Sofia : Imir, 1997. Voir également Kiel (Machiel), « La diffusion de l'islam dans les campagnes bulgares à l'époque ottomane (XV'ème-XIX ${ }^{\text {ème }}$ siècles) ", Revue d'étude 
du monde musulman et de la Méditerranée, 66 (4), 1992, ainsi que Balivet (Michel), « Aux origines de l'islamisation des Balkans ottomans ", Revue d'étude du monde musulman et de la Méditerranée, 66 (4), 1992.

4. Pour plus de détails, voir notamment Nouzille (Jean), «La Bulgarie et les Bulgares après le recensement du 4 décembre 1992 ", Revue d'Europe centrale, 4 (1), 1996, pp. 26-27.

5. Chiffres cités dans Konstantinov (Yulian), "Nation-state" and "Minority" Types of Discourse. Problems of Communication between the Majority and the Islamic Minorities in Contemporary Bulgaria », Innovation, 5(3), 1992, p. 80.

6. On remarquera que ce tableau n'établit pas de distinction entre les Bulgares de confession orthodoxe et les Bulgares de religion musulmane (Pomaks), ce qui tend à pousser les chiffres à la baisse dans la mesure où les Pomaks vivent souvent dans des villages de montagne en communautés relativement isolées. Le critère d'identification retenu est ainsi celui de la "nationalité".

7. Il ne faut toutefois pas attendre davantage de ces données que ce qu'elles peuvent nous offrir (quantitatives d'abord, problème de la qualité des réponses, problème du type et de la méthode d'administration). Elles ne permettent en effet pas de tester les dynamiques à l'œuvre chez les jeunes affichant une foi profonde, ni, partant, de mesurer une éventuelle renaissance de l'islam dans les catégories socialement marginalisées de la population.

8. Voir Georgiev (Jivko) et al., "Njakoi rezultati ot izsledvaneto "Etnokulturnata situacija v Bălgarija, 1992" " (Quelques résultats de l'enquête "la situation ethnoculturelle en Bulgarie, 1992"), Sociologičeski pregled, (3), 1993, pp. 58-59.

9. Entretien avec Ibrahim Ialumov, Sofia, 17 novembre 1997.

10. Voir Georgiev (Jivko) et al., art.cit., p. 55. Notons toutefois qu'environ un tiers des Turcs bulgares ont déclaré parler également bulgare chez eux.

11. Chiffres cités dans Rezultati ot prebrjavaneto na naselenieto (t.1. Demografski harakteristiki), Sofia : Nacionalen statističeski institut, 1994, p. 194.

12. Tous mes remerciements à Nathalie Clayer pour ses précieuses remarques sur les Alévis de Bulgarie.

13. I. Mélikoff propose des observations très stimulantes sur les Kizilbashi du nord-est de la Bulgarie dans Mélikoff (Irène), «La communauté kizilbash du Deli Orman, en Bulgarie », Revue des études islamiques, 60, 1992. On pourra également se reporter à Zarcone (Thierry), « Nouvelles perspectives dans les recherches sur les kizilbas-alévis et les bektachis de la Dobroudja, du Deliorman et de la Thrace orientale ", Anatolia Moderna, (4), 1992.

14. Pour plus de détails, voir Lory (Bernard), «Essai d'inventaire des lieux de culte bektachis en Bulgarie ", Revue des études islamiques, 60, 1992.

15. Interview avec l'enquêteur spécialiste des questions ethniques, Ilia Atanassov, Sofia, 15 novembre 1997.

16. Chiffre cité par Poulton (Hugh), The Balkans. Minorities and States in Conflict, London: Minority Rights Group, 1991. Y. Konstantinov parle de 270000 Pomaks dans Konstantinov (Yulian), art.cit., p. 80. Selon les résultats préliminaires du recensement de 1992, 163735 Bulgares (critère linguistique) et 142938 Bulgares (critère de la nationalité) auraient déclaré professer l'islam. À ce propos, voir Eminov (Ali), Turkish and Other Muslim Minorities, London : Hurst \& Co., 1997, p. 101.

17. Voir Konstantinov (Yulian), art.cit., p. 85.

18. Une bonne présentation de l'histoire des Pomaks depuis l'indépendance pourra être trouvée dans Panajotova (Boriana), «Bălgari, mohamedani i hristijani v Centralnite Rodopi. Pogled vărhu tehnite vzaimootnosenija» (Bulgares, mohamédans et chrétiens dans les Rhodopes du centre. Un regard sur leur situation), in Aspekti na etnokulturnata situacija v Bălgariâ i na Balkanite (Aspects de la situation ethnoculturelle en Bulgarie et dans les Balkans), Sofia: Center for the Study of Democracy \& Fondation Friedrich Nauman, 1992. 
19. Sur les polémiques concernant les origines et l'appartenance nationale des Pomaks, voir Eminov (Ali), op.cit., pp. 99-111.

20. Voir notamment Konstantinov (Yulian), «An Account of Pomak Renaming in Bulgaria (1912-1990) ", contribution à la conférence The Minority Question in the Countries of the Danube Basin, 1918-1990, Dubrovnik, 8-14 avril 1991 (manuscrit) ; Zhelyazkova (Antonina), Bulgaria in Transition. The Muslim minorities, Sofia, 1994, manuscrit, pp. 6-7.

21. L'étymologie du nom fait encore l'objet d'interprétations conflictuelles, autour desquelles se joue notamment la question des origines des Bulgares musulmans. Selon certaines versions, pomak viendrait de "pomamvam / pomamil se", littéralement "celui qui a changé sa foi". Pour d'autres, il renverrait plutôt à "pomagač / pomagal na turcite", c'est-à-dire "celui qui a aidé les Turcs". Voir Panajotova (Boriana), art.cit., p. 35.

22. Chiffres cités par Ivanov (Mihail), Tomova (Ilona), "Ethnic Groups and Interethnic Relations in Bulgaria ", contribution au séminaire bulgaro-allemand Le journalisme professionnel en Europe : les expériences bulgare et allemande, Bankia, 26-30 août 1993, pp. 5-6 (manuscrit).

23. Des phénomènes de rechristianisation seraient d'ailleurs observés depuis quelques années, surtout dans la région de Zlatograd (Benkovski).

24. Voir Georgiev (Jivko) et al., art.cit., p. 55.

25. Voir Konstantinov (Yulian), Alhaug (Gulbrand), Igla (Birgit), « Names of the Bulgarian Pomaks », Nordlyd, (17), 1991, p. 46, cité in Eminov (Ali), op.cit., pp. 108-109.

26. Voir Georgiev (Jivko) et al., art.cit., p. 56.

27. Soulignons à ce propos que, lors de l'expulsion massive des Turcs au début des années 1950, certains Pomaks étaient parvenus à passer la frontière, de sorte qu'existe à l'heure actuelle en Turquie une petite communauté pomaque, qui peut éventuellement faciliter l'installation de ses frères.

28. Selon des sources du ministère de l'Intérieur, en 1989 il y aurait 576927 Tsiganes en Bulgarie, représentant environ 6,4\% de la population (cité in Eminov (Ali), op.cit., p. 112). Ilona Tomova propose une fourchette entre 577000 et 600000 , qui correspond au nombre de Tsiganes identifiés comme tels par les populations voisines (Tomova (Ilona), The Gypsies in the Transition Period, Sofia : International Center for Minority Studies and Intercultural Relations, 1995, p. 13.). Les spécialistes de questions tsiganes Elena Marushiakova et Vesselin Popov considèrent quant à eux que le chiffre de 800000 fournit une estimation plus proche de la réalité (Marušjakova (Elena), Popov (Veselin), Ciganite v Bălgarija (Les Tsiganes en Bulgarie), Sofia : Club 90, 1993, p. 95).

29. Réciproquement un faible pourcentage des chrétiens se déclare Bulgare "ethnique".

30. Sur l'histoire des Tsiganes de Bulgarie, voir Crowe (David), "Bulgaria ", in A History of the Gypsies of Eastern Europe and Russia, New York : Saint Martin's Griffin, 1996.

31. Voir Tomova (Ilona), The Gypsies (op.cit.), p. 71.

32. Voir ibid., pp. 28-29.

33. Voir Konstantinov (Yulian), «Hunting for Gaps Through Boundaries: Gypsy Tactics for Economic Survival in the Context of the Second Phase of Post-Totalitarian Changes in Bulgaria (1994-...) », Innovation, 7 (3), 1994.

34. Voir Tomova (Ilona), The Gypsies (op.cit.), p. 31.

35. Voir ibid., p. 19.

36. Voir Iltchev (Ivan), Perry (Duncan), « Bulgarian Ethnic Groups : Politics and Perceptions », RFE/RL Research Report, 2 (12), 1991.

37. Voir Marušjakova (Elena), Popov (Veselin), op.cit., pp. 109-127.

38. Voir Tomova (Ilona), The Gypsies (op.cit.), p. 21.

39. En juin 1990, la dette extérieure de la Bulgarie s'élevait à plus de 10 milliards de dollars en juin 1990, soit le ratio par habitant (1 200 dollars/hab.) le plus élevé de tout le bloc socialiste. 
Chiffres cités par Lhomel (Edith), « Bulgarie : de lourds handicaps », Transitions économiques à l'Est (1989-1995), Paris : La Documentation française, 1996, p. 129.

40. Un excellent bilan de la transition économique bulgare peut être trouvé dans ibid., pp. 127-136. On se reportera également au très complet Études de l'OCDE, La Bulgarie, Paris, mars 1997.

41. Chiffres cités dans ibid., p. 39.

42. Sur les déboires de la politique agricole bulgare, on pourra consulter Blotnicki (Laurence), "Les avancées contrariées de la restructuration agricole en Bulgarie», Revue d'Etudes Comparatives Est-Ouest, (3), septembre 1995.

43. Chiffres cités dans Études de l'OCDE, op.cit., p. 132.

44. Le propos vaut a fortiori dans le cas des Tsiganes dont $93 \%$ sont « restés en dehors du processus de restitution des terres en Bulgarie, parce qu'ils n'en possèdent pas ». Voir Mitev (Petar-Emil), ed., Relations of Compatibility and Incompatibility in the Everyday Life of Christians and Muslims in Bulgaria, Sofia: International Centre for Minority Studies and Intercultural Relations, 1994, p. 204.

45. Pour plus de détails sur les situations de Kardjali et Razgrad, voir Anagnostou (Dia), «Regional and Post-Communist Restructuring in Greece and Bulgaria: Socioeconomic Change and Muslim Identity ", présentation à la $92^{\text {ème }}$ rencontre annuelle de l'Association américaine de science politique, 29 août- ${ }^{\mathrm{er}}$ septembre 1996, San Francisco, pp. 22-23 (manuscrit).

46. Pour de plus amples détails, voir Hristov (Mihajl), «Fenomenite na bălgarskoto tjutjunevo stopanstvo » (Les phénomènes relatifs à l'économie du tabac bulgare), Sega, (25), 3-9/07/97.

47. Voir Anagnostou (Dia), art.cit., p. 23.

48. Voir ibid., p. 24.

49. Voir ibid., p. 32.

50. Voir ibid., p. 33.

51. Le propos vaut essentiellement pour le nord-est de la Bulgarie. Voir Vicheva (Tsena), "Deliorman. Changes and Problems in the Relations between Ethnic Communities ", in Relations of Compatibility and Incompatibility (op.cit.), pp. 375-378.

52. Voir Vicheva (Tsena), art.cit., p. 378.

53. La remarque vaut également pour les Pomaks, dont une partie utilise des noms slaves afin d'obtenir plus facilement un emploi saisonnier en Grèce.

\section{RÉSUMÉS}

Par la présentation des différentes communautés islamiques de Bulgarie, l'auteur met souligne les relations ambiguës qu'entretiennent marqueurs ethniques et religieux et pointe les glissements s'opérant des uns aux autres dans le cadre de stratégies d'intégration aux groupes nationaux plus nombreux.

\section{AUTEUR}

\section{NADĖGE RAGARU}

Nadège Ragaru est doctorante à l'IEP de Paris. 